\title{
Consensus interpretation of the p.Met34Thr and p.Val37lle variants in GJB2 by the ClinGen Hearing Loss Expert Panel
}

\author{
Jun Shen, PhD, FACMG $\oplus^{1,2,3}$, Andrea M. Oza, MS, CGC $\odot^{3,4}$, Ignacio del Castillo, PhD $\oplus^{5,6}$, \\ Hatice Duzkale, MD, PhD $\oplus^{7}$, Tatsuo Matsunaga, $\mathrm{MD}, \mathrm{PhD}{ }^{8}{ }^{8}$, Arti Pandya, $\mathrm{MD}^{9}$, \\ Hyunseok P. Kang, MD $\odot^{10}$, Rebecca Mar-Heyming, PhD ${ }^{10}{ }^{10}$, Saurav Guha, PhD, FACMG ${ }^{10,38}$, \\ Krista Moyer, MS, $\mathrm{CGC}^{10}$, Christine Lo, MS ${ }^{10}{ }^{10}$, Margaret Kenna, MD ${ }^{2,4}$, \\ John J. Alexander, PhD, FACMG ${ }^{11,39}$, Yan Zhang, MD ${ }^{12}$, Yoel Hirsch, BS ${ }^{13}$, \\ Minjie Luo, PhD, FACMG ${ }^{14,15}$, Ye Cao, PhD ${ }^{16}$, Kwong Wai Choy, PhD ${ }^{16}$, \\ Yen-Fu Cheng, MD, PhD ${ }^{17,18,19}$, Karen B. Avraham, $\mathrm{PhD}{ }^{20}$, Xinhua $\mathrm{Hu}, \mathrm{PhD}{ }^{24}$, \\ Gema Garrido, BS ${ }^{5,6}$, Miguel A. Moreno-Pelayo, PhD ${ }^{5,6}$, John Greinwald, $\mathrm{MD}^{7}$, \\ Kejian Zhang, MD, FACMG ${ }^{7}$, Yukun Zeng, MD ${ }^{12}$, Zippora Brownstein, PhD ${ }^{20}$, \\ Lina Basel-Salmon, MD, PhD ${ }^{20,21,22,23}$, Bella Davidov, MS ${ }^{20}$, Moshe Frydman, MD ${ }^{20,25}$, \\ Tzvi Weiden, $\mathrm{BS}^{26}$, Narasimhan Nagan, PhD, FACMG ${ }^{27}$, Alecia Willis, PhD, FACMG ${ }^{28}$, \\ Sarah E. Hemphill, BS ${ }^{3}$, Andrew R. Grant, BS ${ }^{3,29}$, Rebecca K. Siegert, BS ${ }^{3,29}$, \\ Marina T. DiStefano, PhD $\oplus^{3}$, Sami S. Amr, PhD, FACMG $\otimes^{1,2,3}$, \\ Heidi L. Rehm, PhD, FACMG $\oplus^{1,2,3,29,30}$ and Ahmad N. Abou Tayoun, PhD, FACMG ${ }^{31}$ \\ on behalf of the ClinGen Hearing Loss Working Group
}

Purpose: Pathogenic variants in GJB2 are the most common cause of autosomal recessive sensorineural hearing loss. The classification of c.101T >C/p.Met34Thr and c.109G $>$ A/p.Val37Ile in GJB2 are controversial. Therefore, an expert consensus is required for the interpretation of these two variants.

Methods: The ClinGen Hearing Loss Expert Panel collected published data and shared unpublished information from contributing laboratories and clinics regarding the two variants. Functional, computational, allelic, and segregation data were also obtained. Case-control statistical analyses were performed.

Results: The panel reviewed the synthesized information, and classified the p.Met34Thr and p.Val37Ile variants utilizing professional variant interpretation guidelines and professional judgment. We found that p.Met34Thr and p.Val37Ile are significantly overrepresented in hearing loss patients, compared with population controls. Individuals homozygous or compound heterozygous for p.Met34Thr or p.Val37Ile typically manifest mild to moderate hearing loss. Several other types of evidence also support pathogenic roles for these two variants.

Conclusion: Resolving controversies in variant classification requires coordinated effort among a panel of international multi-institutional experts to share data, standardize classification guidelines, review evidence, and reach a consensus. We concluded that p.Met34Thr and p.Val37lle variants in GJB2 are pathogenic for autosomal recessive nonsyndromic hearing loss with variable expressivity and incomplete penetrance.

Genetics in Medicine (2019) 21:2442-2452; https://doi.org/10.1038/s41436019-0535-9

Keywords: ClinGen; hearing loss; incomplete penetrance; variant classification; variant interpretation

\section{INTRODUCTION}

Variants with incomplete penetrance for Mendelian conditions pose significant challenges for clinical interpretation because they are relatively common in the population and present in healthy individuals. ${ }^{1}$ No such variants have been rigorously reviewed and classified according to the American College of Medical Genetics and Genomics and the Association for Molecular Pathology (ACMG/AMP) guidelines. ${ }^{2}$ To demonstrate the best practice to interpret such variants, the
ClinGen Hearing Loss Expert Panel (HL-EP) applied hearing loss gene-specific ACMG/AMP guidelines ${ }^{3}$ to interpret two controversial variants in GJB2.

GJB2 encodes connexin 26, a member of the gap junction protein family. Gap junctions are intercellular channels allowing the coupling of adjacent cells to share molecules, ions, and electrical signals. Each gap junction channel is composed of two connected hemichannels called connexons, one on either membrane of neighboring cells. Each connexon

Correspondence: Jun Shen (jshen5@bwh.harvard.edu) or Ahmad N. Abou Tayoun (Ahmad.Tayoun@ajch.ae). ${ }^{*}$ Affiliations are listed at the end of the paper. 
is a hexamer of the same or different connexin units. Biallelic pathogenic variants in GJB2 (NM_004004.5) are the most frequently identified cause of autosomal recessive sensorineural hearing loss. ${ }^{4,5}$ Hundreds of GJB2 variants have been reported in patients with hearing loss. Premature termination codons (PTC), such as c.35delG, c.167delT, and c.235delC common in European, Ashkenazi Jewish, and Asian populations respectively, are established pathogenic variants. However, classifications of two notable missense variants, c.101T $>$ C/p.Met34Thr and c.109G $>$ A/p.Val37lle, have been controversial. P.Met34Thr was first reported as being associated with dominant hearing loss, ${ }^{4}$ but its pathogenicity for dominant hearing loss was later challenged because of subsequent identification of its occurrence in individuals with normal hearing, ${ }^{5,6}$ and an autosomal recessive mode of inheritance was suggested. ${ }^{7-9}$ P.Val37lle was first identified as a polymorphism in a heterozygous control, ${ }^{10}$ and later found to be homozygous or in trans with known pathogenic GJB2 variants in affected individuals. ${ }^{7,11}$ Both variants were found relatively frequently in the general population. Some homozygotes for these variants appeared to have normal hearing. ${ }^{12,13}$ Reduced penetrance was proposed to explain the inconsistency. ${ }^{14}$ We surveyed clinical laboratories in the United States and Canada regarding the classification of these variants and found significant variability across different laboratories (Supplementary Information). Therefore, we considered it a priority to resolve the controversy and reach a consensus.

Herein, we report the ClinGen and ClinVar effort to resolve controversies regarding p.Met34Thr and p.Val37Ile classification assertions and demonstrate the best practice to interpret variants with incomplete penetrance.

\section{MATERIALS AND METHODS ClinGen Hearing Loss Expert Panel}

The ClinGen HL-EP includes otolaryngologists caring for patients with hereditary hearing loss, medical geneticists, clinical laboratory diagnosticians, molecular pathologists, genetic counselors, and investigators specialized in auditory research. ${ }^{3}$ This group was convened to develop specifications of the ACMG/AMP guidelines for interpreting sequence variants in genes associated to hearing loss. ${ }^{3}$

\section{Data sources}

Fifteen genetic testing laboratories and clinics contributed data to this study (Table 1). Individuals tested for p.Met34Thr or p.Val37Ile were designated as cases if hearing loss was the indication or as population controls if carrier screening was the indication. Counsyl data were solely based on carrier screening, thus representing the general population. We also considered information from the Genome Aggregation Database (gnomAD; http://gnomad.broadinstitute.org/) as population controls. Testing methods included specific variant testing, GJB2 sequencing, large multigene panels, and exome sequencing. Individuals from sites with unspecified total numbers tested and relatives of probands were excluded from statistical analyses. Data collected included total number of individuals tested for p.Met34Thr or p. Val37Ile in GJB2, ethnicities, allele states, and phenotypic information (age at onset of hearing loss, age at testing, type of hearing loss, severity, laterality, frequency range affected, family history, and other clinical features). The study was approved by respective Institutional Review Boards, Helsinki Committees, or equivalent ethics committees of participating institutions involving research on human subjects.

\section{Variant interpretation}

Data were analyzed and interpreted according to ACMG/ AMP guidelines ${ }^{2}$ and hearing loss gene-specific criteria by the ClinGen HL-EP. ${ }^{3}$ Original evidence codes included a first letter P ("pathogenic") or B ("benign"), followed by VS ("very strong"), S ("strong"), M ("moderate"), P ("supporting"), or A ("stand-alone") to indicate the strength level and a category number. ${ }^{2}$ Modified codes included a suffix of an underscore and the adjusted strength level. ${ }^{3}$

\section{Statistical analysis}

For $2 \times 2$ contingency tables, odds ratios (OR), 95\% confidence intervals (CI), $Z$ statistics, and $p$ values were calculated using MEDCALC (https://www.medcalc.org/calc/ odds_ratio.php). Four types of case-population comparisons were performed: (1) homozygotes in overall populations, (2) homozygotes in ethnicity-stratified populations, (3) alleles in ethnicity-stratified populations, and (4) homozygotes and compound heterozygotes in ethnicity-stratified populations. GnomAD data were included except for analyses involving compound heterozygotes, where only Counsyl data were used as population controls, because individual data were unavailable in gnomAD. Compound heterozygosity was presumed in individuals with two variants in GJB2 never reported to have occurred in cis.

For comparisons of the severity among different genotype groups, an ordinal logistic regression model was used. No covariates such as age and sex were included, because the information was not available for all individuals. The proportions of the sample sizes of the genotype groups were used as weights for the analysis. Type 1 test was used to test the overall significant difference among all groups and ad hoc analysis was performed to compare the estimated odds ratio between each pair of specific gene types. The statistical significance was defined as $p$ value $<0.05$. The analysis was performed with SAS software (version 9.4).

\section{RESULTS}

\section{Case-control evidence}

P.Met34Thr and p.Val37Ile have been reported in patients with hearing loss in various populations (Supplementary Information). To perform accurate case-control comparisons, the HL-EP decided not to rely on published cases in the literature due to concerns of the publication bias. Instead, we obtained data from 15 contributing sites (Table 1). The level of details provided by each site varied. Three sites $(\mathrm{BCH}, \mathrm{DY}$, 


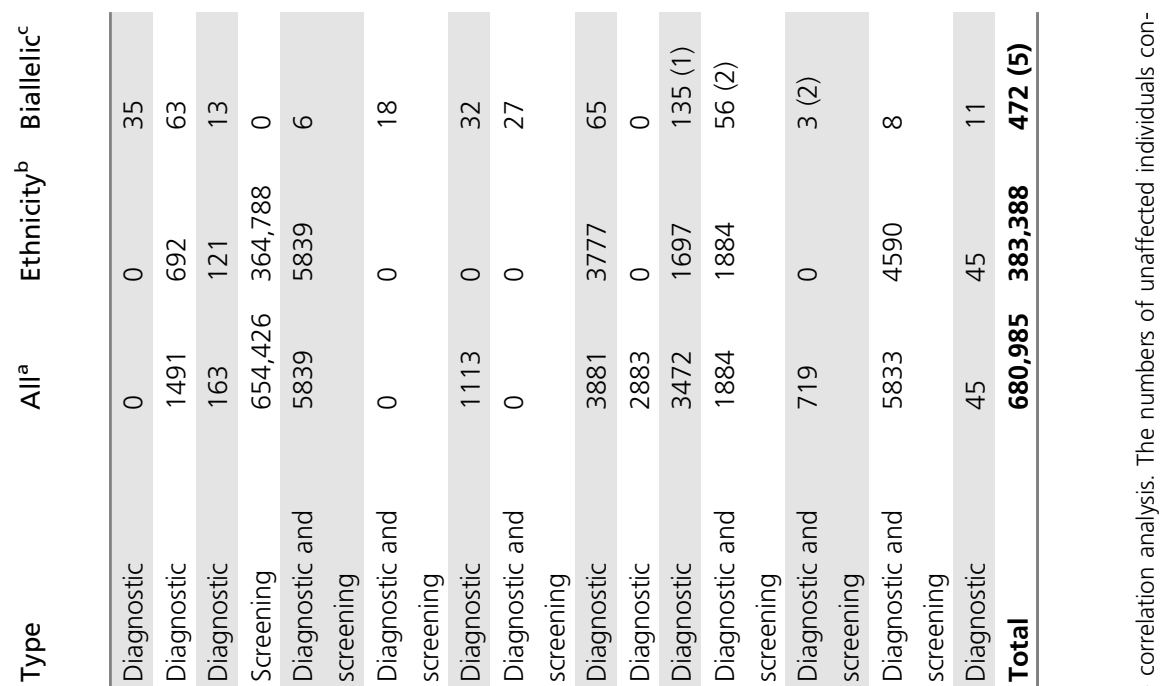

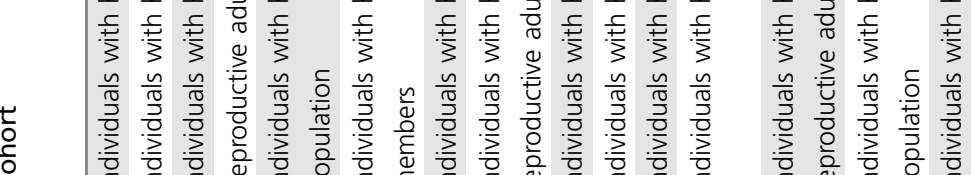

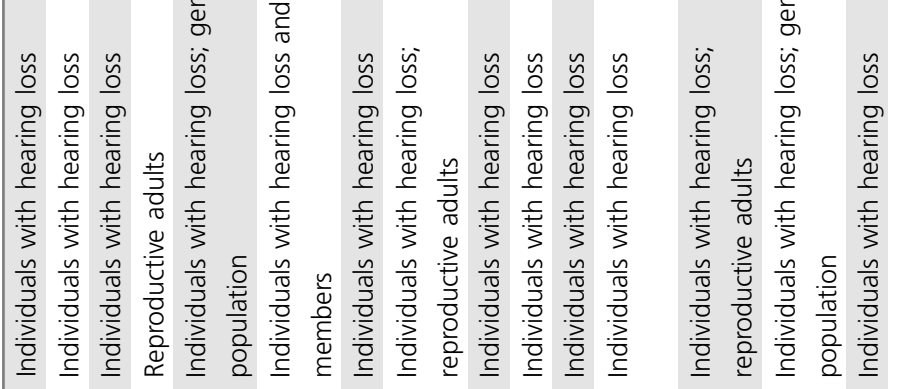

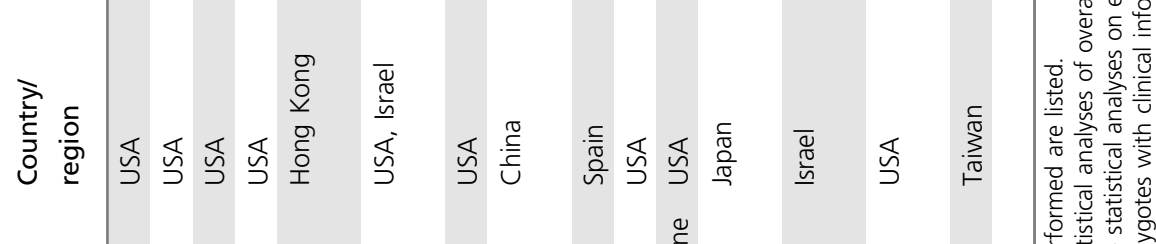

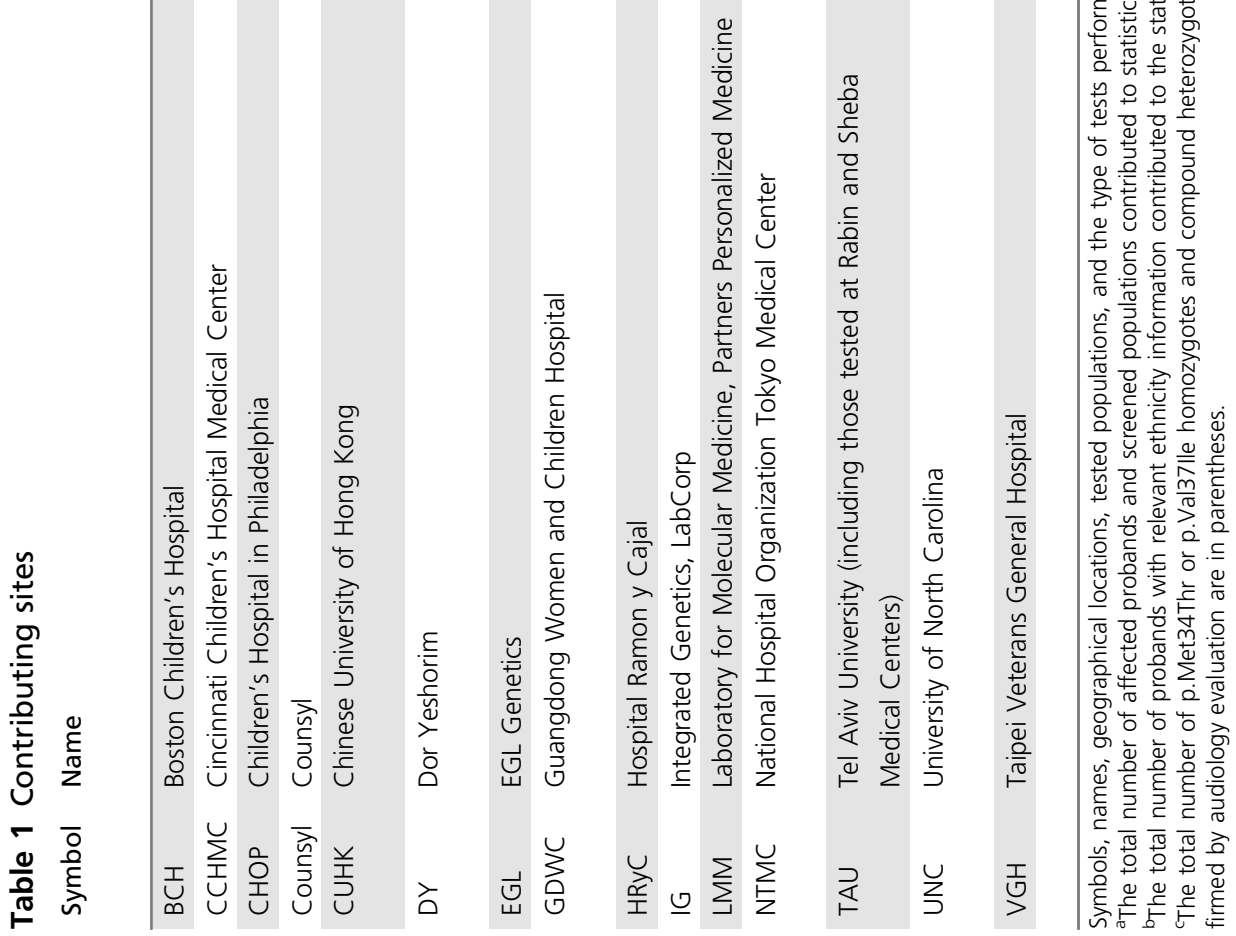


Table 2 Summary statistics for p.Met34Thr

\begin{tabular}{|c|c|c|c|c|c|c|c|}
\hline All ${ }^{a}$ & Cases $^{\mathrm{b}}$ & Population $^{c}$ & Counsyl & OR & $95 \% \mathrm{Cl}$ & $Z$ & $P$ value \\
\hline Total number of alleles tested for GJB2 & 35,270 & $1,604,678$ & $1,308,852$ & & & & \\
\hline Total number of p.Met34Thr heterozygotes & 362 & 10,754 & 8282 & & & & \\
\hline Total number of p.Met34Thr homozygotes & 29 & 81 & 54 & 16 & $11-25$ & 13 & $<0.0001$ \\
\hline Total number of p.Met34Thr compound heterozygotes ${ }^{d}$ & 147 & $N A^{e}$ & 135 & & & & \\
\hline Overall p.Met34Thr allele frequency & 0.0119 & 0.0068 & 0.0064 & & & & \\
\hline Number of European individuals tested for GJB2 & 7962 & 382,842 & 304,433 & & & & \\
\hline Number of European alleles tested for GJB2 & 15,924 & 765,684 & 608,866 & & & & \\
\hline Number of European individuals with p.Met34Thr & 207 & 7915 & 5769 & & & & \\
\hline Number of European p.Met34Thr heterozygotes & 193 & 7846 & 5725 & & & & \\
\hline p.Met34Thr allele frequency in Europeans & 0.0139 & 0.0104 & 0.0095 & & & & \\
\hline
\end{tabular}

Cl confidence interval, OR odds ratio.

Bold values indicate odds ratio exceeding 5 to meet PS4, as specified by the HL ClinGen group.

${ }^{a}$ Only probands (unrelated individuals) were counted. However, we could not rule out the possibility of related cases from different sites because cases were de-identified before being shared. Nevertheless, the likelihood of such occurrence would be low and would not significantly impact the conclusion.

${ }^{b}$ The total number of cases included in statistical analyses did not include BCH, DY, and GDWC where the total number of individuals tested at these sites were not available.

'The total population data were from Counsyl, CUHK, TAU, UNC, and gnomAD.

${ }^{\mathrm{d}}$ Compound heterozygosity was presumed in individuals with a second pathogenic or likely pathogenic variant in GJB2 that had never been reported to have occurred in cis.

eNA: Not available, because individual allele state information is not available from gnomAD.

${ }^{f}$ Analyses involving compound heterozygotes were performed using Counsyl data as the population control (see "Materials and Methods").

and GDWC) reported only cases with biallelic GJB2 variants involving p.Met34Thr or p.Val37Ile. Eleven sites reported results from 17,635 probands with hearing loss tested for these two variants. Ethnicity information was available from eight sites on 7962 European probands and 2066 Asian probands. Five sites (Counsyl, CUHK, NTMC, TAU, and UNC) provided population screening data from 664,114 individuals, including 306,982 Europeans and 66,423 Asians (Tables 2 and 3).

We compared frequencies of individuals with these two variants between cases in our multicenter cohort and population controls. P.Met34Thr and p.Val37lle homozygotes are significantly enriched in cases with ORs of $16(95 \%$ CI $11-25, Z=13, p<0.0001$ ) (Table 2) and 20 (95\% CI 17-24, $Z$ $=31, p<0.0001)$ (Table 3 ) respectively.

Because the allele frequencies of p.Met34Thr and p.Val37Ile were observed highest in European and East Asian populations, respectively, we performed statistical analyses on stratified subpopulations by ethnicity to remove the confounding factor of different ethnic compositions between cases and controls. The significance still held when we performed ethnicity-specific analysis. P.Met34Thr homozygotes were significantly enriched in cases over population controls in Europeans $(\mathrm{OR}=9.8,95 \% \mathrm{CI}=5.5-17, Z=7.8, p$ $<0.0001$ ) (Table 2) and p.Val37Ile homozygotes in Asians $(\mathrm{OR}=12,95 \% \mathrm{CI}=9.1-15, Z=19, p<0.0001)$ (Table 3), respectively. When we considered all biallelic cases involving
p.Met34Thr or p.Val37Ile, including homozygotes and compound heterozygotes with another pathogenic variant in GJB2, the enrichments were more significant with ORs of 29 (95\% CI 22-37, $Z=25, p<0.0001$ ) for p.Met34Thr in Europeans (Table 2) and of 19 (95\% CI 15-24, $Z=26, p<$ 0.0001) for p.Val37Ile in Asians (Table 3) in cases with hearing loss in our multicenter cohort over those who underwent carrier screening at Counsyl. Therefore, both variants meet PS4 (prevalence in affecteds statistically increased over controls) according to ACMG/AMP guidelines. $^{2}$

Although alleles were statistically enriched in cases in our multicenter cohort over the general population with ORs of 1.3 (95\% CI 1.2-1.5, $Z=4.2, p<0.0001$ ) for p.Met34Thr in Europeans (Table 2) and of 1.7 (95\% CI 1.5-1.9, $Z=8.1, p<$ 0.0001) for p.Val37Ile in Asians (Table 3), the ORs did not exceed 5 to satisfy PS4. We concluded that comparing genotype frequencies is more appropriate than comparing allele frequencies in case-control analyses to interpret variants associated with an autosomal recessive condition, because the unaffected heterozygous carriers contributed the majority of alleles.

\section{Computational predictions}

The REVEL scores were 0.702 and 0.657 for p.Met34Thr and p.Val37Ile, respectively. PP3 (computational predictions support pathogenicity) could be applied to p.Met34Thr 
Table 3 Summary statistics for p.Val37lle

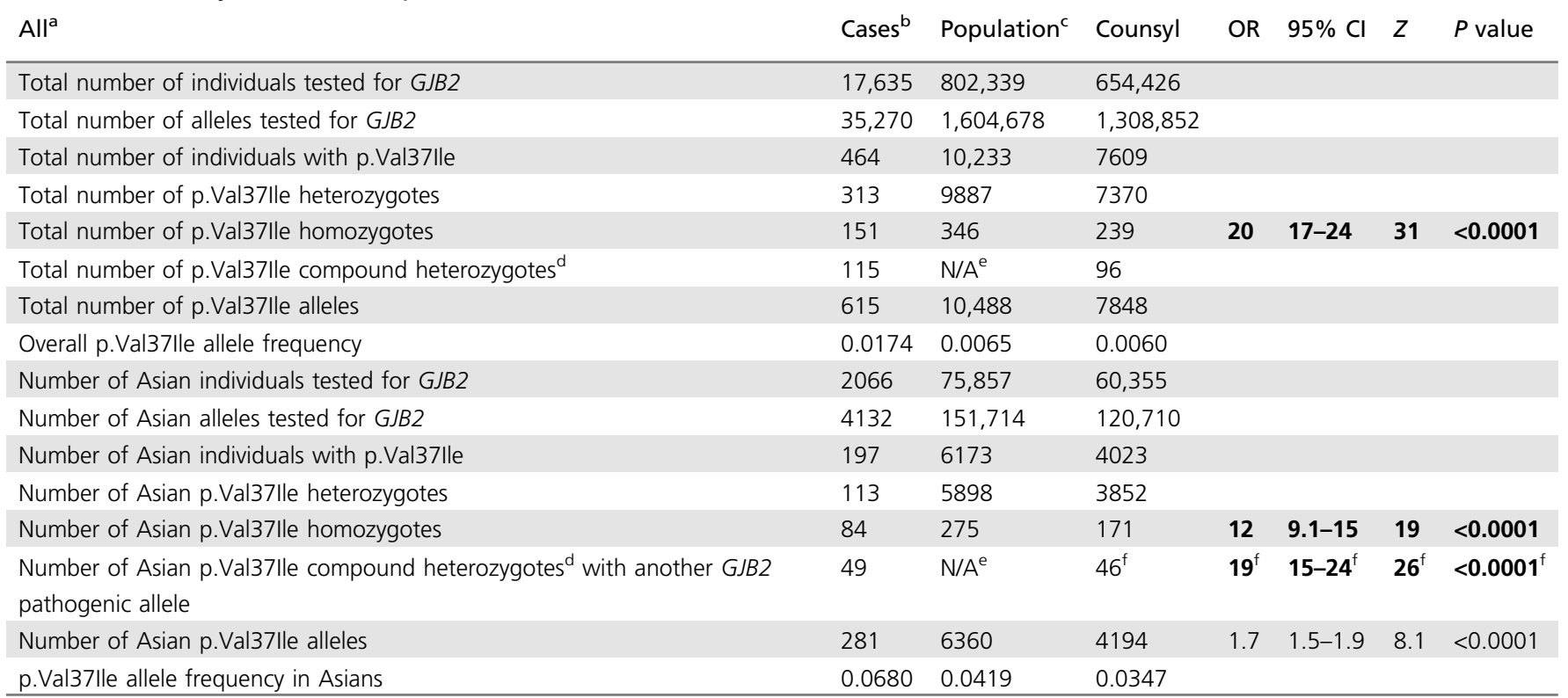

Cl confidence interval, OR odds ratio.

Bold values indicate odds ratio exceeding 5 to meet PS4, as specified by the HL ClinGen group.

${ }^{a}$ Only probands (unrelated individuals) were counted. However, we could not rule out the possibility of related cases from different sites because cases were de-identified before being shared. Nevertheless, the likelihood of such occurrence would be low and would not significantly impact the conclusion.

${ }^{\mathrm{b}}$ The total number of cases included in statistical analyses did not include BCH, DY, and GDWC where the total number of individuals tested at these sites were not available.

'The total population data were from Counsyl, CUHK, TAU, UNC, and gnomAD.

${ }^{\mathrm{d} C}$ Compound heterozygosity was presumed in individuals with a second pathogenic or likely pathogenic variant in GJB2 that had never been reported to have occurred in cis.

eNA: Not available, because individual allele state information is not available from gnomAD.

${ }^{f}$ Analyses involving compound heterozygotes were performed using Counsyl data as the population control (see "Materials and Methods").

(REVEL $\geq 0.7$ ) but not to p.Val37lle because its REVEL score did not reach the threshold (0.7) to support pathogenicity but exceeded the ceiling (0.15) to support a benign role as specified by the ClinGen HL-EP. ${ }^{3}$

Different amino acid changes at positions p.Met34 and p. Val37 have been reported in hearing loss patients (Supplementary Information). While they do not constitute sufficient evidence for PM5 (different change at the same residue as a known pathogenic variant), multiple variants of uncertain significance leaning toward pathogenicity affecting the same amino acid residues corroborate with each other.

\section{Segregation evidence}

Literature review showed that both variants segregated with hearing loss in families. Furthermore, we reported at least 35 affected siblings with the same homozygous or compound heterozygous genotypes as probands, including 16 with $\mathrm{p}$. Met34Thr and 21 with p.Val37Ile (Table S2). Therefore, both variants meet PP1_Strong (cosegregation in families, modified to strong). ${ }^{2,3}$

P.Met34Thr and p.Val37Ile homozygotes were present in large population databases and identified by population carrier screening. However, clinical information was unavailable. Some individuals may be underdiagnosed. Furthermore, it has been reported that p.Val37Ile homozygotes lose hearing at $\sim 1 \mathrm{~dB} /$ year, ${ }^{15}$ implicating an age-dependent penetrance. The penetrance by young adulthood was estimated to be $17 \%$ (ref. ${ }^{16}$ ). Therefore, we did not consider hearing individuals with biallelic p.Met34Thr or p.Val37lle as observations in controls or nonsegregation.

\section{Allelic evidence}

Both variants have been identified in affected homozygotes or compound heterozygotes. In our multicenter cohort, we observed 138 p.Met34Thr and 141 p.Val37lle compound heterozygotes (Table S2). However, because of high population allele frequencies and large sample sizes, we applied PM3 (in trans with a pathogenic variant in an affected individual) without modifying to strong despite the large number of biallelic cases identified.

\section{Functional evidence}

P.Met34Thr altered gap junction function in Xenopus oocytes $^{17-19}$ and mammalian cells. ${ }^{20-22}$ It had a similar subcellular localization pattern as wild-type connexin 26 in human sweat glands, in transfected HeLa cells, and in transfected COS-7 cells. ${ }^{18,20,21}$ Paired Xenopus oocytes showed robust conductance when injected with in vitro transcribed human wild-type GJB2 messenger RNA (mRNA), reduced conductance when coinjected with wild-type and variant mRNA, and no coupling conductance above 
background when injected with variant mRNA only. ${ }^{17-19}$ Coinjection of GJB2 c.101T>C with wild-type connexin 30 (encoded by GJB6) or connexin 31 (encoded by GJB3) also showed reduced conductance. ${ }^{19}$ Single channel conductance in $\mathrm{c} .101 \mathrm{~T}>\mathrm{C}$ transfected HeLa cells was reduced, ${ }^{22}$ as supported by molecular dynamics simulations. ${ }^{23}$ Furthermore, c.101T $>\mathrm{C}$ transfected HeLa cells did not transfer Lucifer yellow (a fluorescent dye) to neighbors across gap junctions as wild-type connexin 26 transfected cells ${ }^{20,22,24}$ and the ability to transfer neurobiotin was also reduced. $^{21}$ However, c.101T $>\mathrm{C}$ transfected HeLa cells were able to load the dye in response to nonphysiological zero extracellular $\mathrm{Ca}^{2+}$ stimulus, ${ }^{25}$ suggesting that p.Met34Thr variant connexin hemichannels may retain some residual function under unusual circumstances. The atomic structure of human connexin 26 gap junction channels revealed that the methyl group in p.Met34 interacts with p.Trp3 in the amino-terminal helix of an adjacent protomer to stabilize the hexameric channel, ${ }^{26}$ and alteration of the residue was predicted to impact gap junction coupling. ${ }^{27}$

Similarly, Xenopus oocytes injected with mRNA encoding $\mathrm{p}$. Val37Ile showed no conductance above background, and coinjection of p.Val37Ile variant connexin 26 with wild-type connexin 26, connexin 30, or connexin 31 showed reduced conductance. ${ }^{19,28}$ Propidium iodide dye transfer was impaired in GJB2 c.109G >A transfected HEK293T cells. ${ }^{29}$ Homozygous knock-in mouse model of c.109G>A in Gjb2 was reported to have progressive mild hearing loss, more pronounced at higher sound frequencies. ${ }^{30}$ However, because the codon for p.Val37 in mouse (GTG) was different from that in human (GTT), c.109G $>$ A in mouse would translate into p.Val37Met, so it was not counted toward strong functional evidence. Nonetheless, this in vivo animal study was consistent with in vitro findings suggesting alteration of p.Val37 impacts GJB2 function.

These functional studies suggest p.Met34Thr and p.Val37Ile impact connexin 26 function; however, they may not truly reflect the biologic process in human cochlea.

\section{Genotype-phenotype correlation}

We obtained clinical information for 472 cases with biallelic GJB2 genotypes involving p.Met34Thr or p.Val37Ile (Table S2). P.Met34Thr was with a PTC in 86 cases, including 73 with c.35delG. P.Val37Ile was with a PTC in 92 cases, including 32 with c.235delC (Table 4). We characterized hearing loss for different genotypes, although information was incomplete and nonuniform. Most cases had hearing loss before 18 years old. It was typically bilateral, mild to moderate, and affecting mid- to high sound frequencies. Progression was reported in all genotype categories (Table 4). In some individuals, profound hearing loss could be due to age-dependent progression or other etiologies. For example, one had meningitis at 1.5 years old, one was 76 years old when tested, and one infant had asymmetric hearing loss with moderate loss in the left ear and severe-to-profound loss in the right ear (Table S2).
Ordered logistic regression models showed no statistically significant difference in severity among p.Met34Thr or p. Val37Ile homozygotes, [p.Met34Thr];[p.Val37Ile], and their corresponding compound heterozygotes with a PTC $(p=$ 0.93), but p.Met34Thr or p.Val37Ile homozygotes or compound heterozygotes with a PTC presented significantly milder hearing loss than a cohort of homozygotes $(p<0.05)$, the majority of whom had profound hearing loss. [p. Met34Thr];[p.Val37Ile] compound heterozygotes also had milder hearing loss than c.35delG homozygotes, but the small sample size precluded statistical significance (Table 4).

One infant with [p.Val37Ile];[c.235delC] passed newborn hearing screening but audiology evaluation detected unilateral mild high-frequency hearing loss at 5 weeks, which progressed to bilateral mild high-frequency hearing loss at 20 weeks. One self-reported unaffected parent was found to be [p.Met34Thr];[c.167delT] via familial testing, and follow-up audiology evaluation revealed mild high-frequency hearing loss at 47 years of age.

Taken together, phenotypic manifestation of GJB2 p. Met34Thr and p.Val37Ile related hearing loss varied from apparently normal to profound. However, these two variants were typically associated with bilateral mild to moderate sensorineural hearing loss, affecting high to mid-sound frequencies. Progression has been reported. There was no significant difference in severity among p.Met34Thr or p. Val37Ile homozygotes and compound heterozygotes with another PTC (Table 4). The penetrance could not be calculated. However, among all individuals with biallelic GJB2 variants involving p.Met34Thr or p.Val37Ile, more compound heterozygotes than homozygotes were observed in our multicenter case cohort compared with the carrier screening population by Counsyl for both p.Met34Thr (OR 2.03, 95\% CI 1.22-3.37, $Z=2.7, p<0.0064)$ and p.Val37Ile (OR 1.90, 95\% CI 1.35-2.66, $Z=3.7, p=0.0002$ ), suggesting a higher penetrance for compound heterozygous genotypes than p.Met34Thr or p.Val37Ile homozygosity.

\section{Summary interpretation}

In summary, p.Met34Thr and p.Val37Ile were classified as pathogenic based on PS4 (homozygotes and compound heterozygotes are significantly enriched in cases over population controls), PP1_Strong (segregated with hearing loss in many affected family members), and PM3 (found in trans with many different pathogenic GJB2 variants in patients with hearing loss).

\section{DISCUSSION}

\section{Interpreting variants using case-control statistics}

Maximum population allele frequencies for p.Met34Thr and p.Val37Ile are $2 \%$ in Finnish and $8 \%$ in East Asian in gnomAD, respectively, and in 3.5\% (7/198) of Finnish and $17 \%(32 / 186)$ of Chinese Dai in Xishuangbanna in the 1000 Genomes Project, respectively. The BA1 (stand-alone for benign, population allele frequency $>5 \%$ ) criterion is not applicable here to p.Val37Ile, because of conflicting evidence 


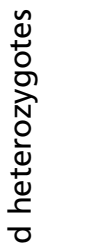

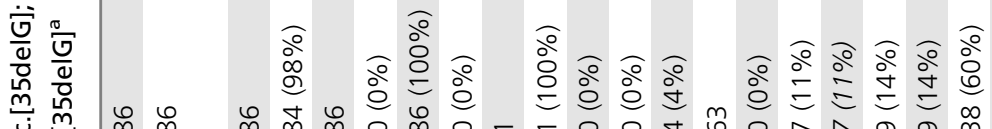

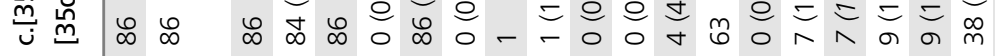

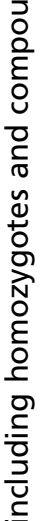

$\frac{\frac{0}{0}}{\overline{\bar{N}}}$

$\stackrel{\ddot{\frac{\Phi}{N}}}{\frac{m}{\sigma}}$

$\stackrel{\frac{m}{\pi}}{\grave{a}}$ $\bar{m} \cong$

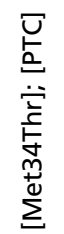

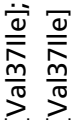

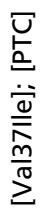

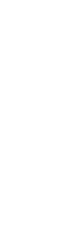

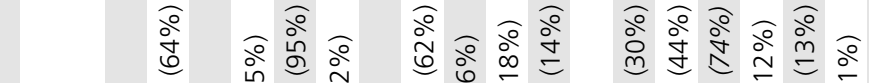

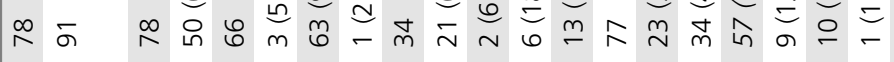

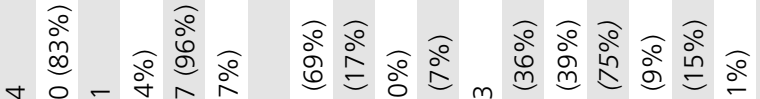

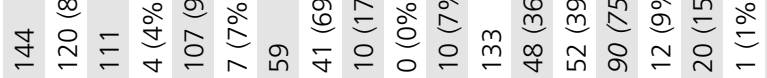

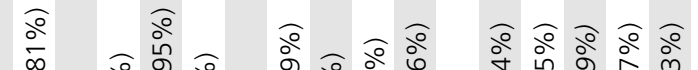

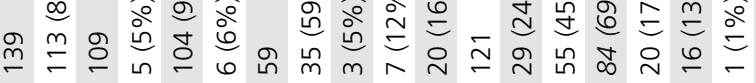

高

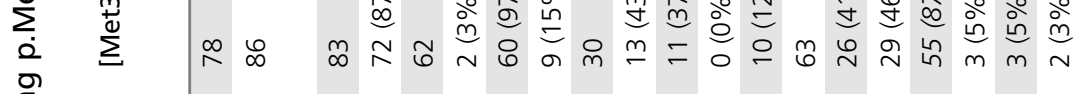

을

ড)

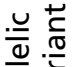

.

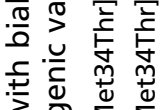

उ

嵌

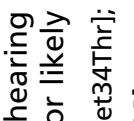

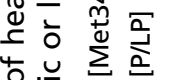

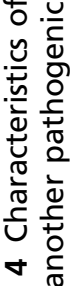

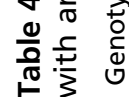

$\stackrel{\infty}{m}$

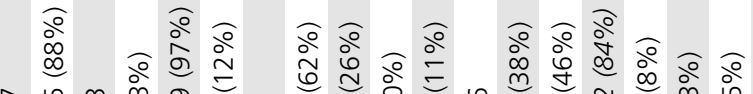

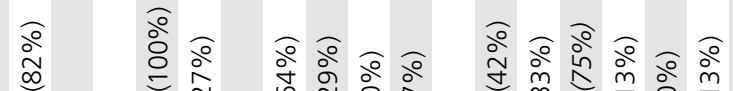

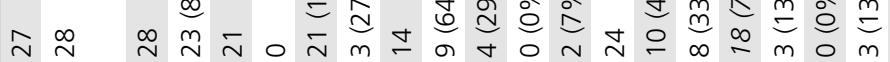
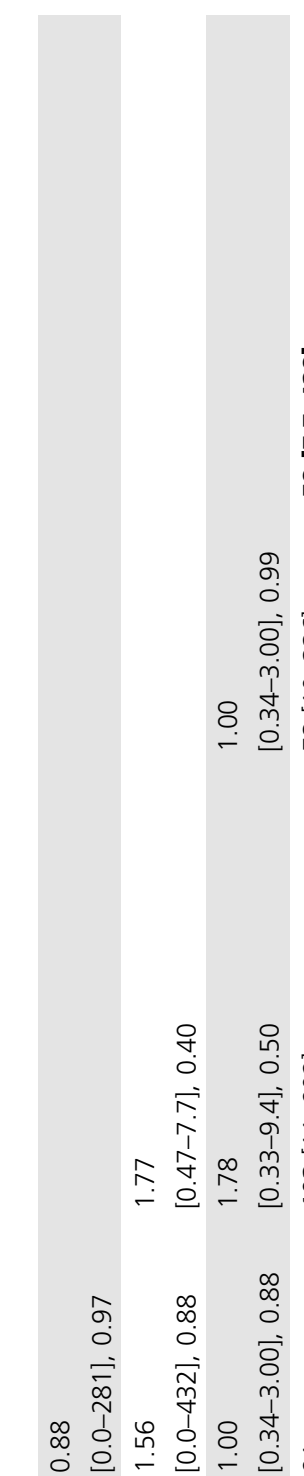

离

co

นึ

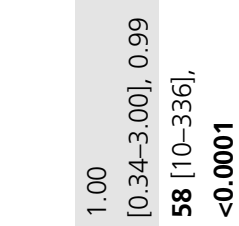

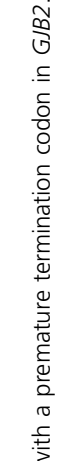

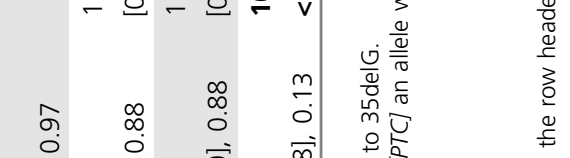

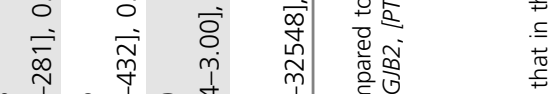

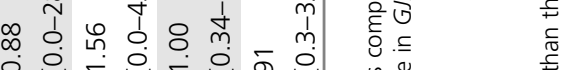

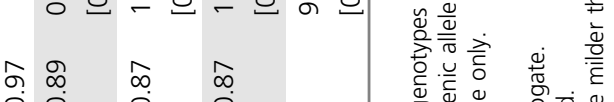

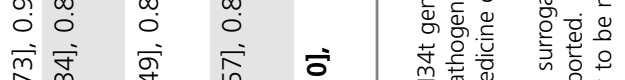

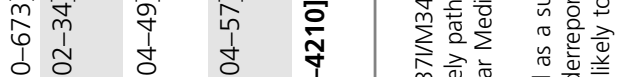

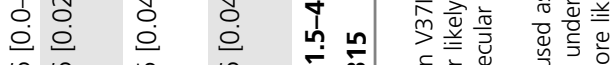

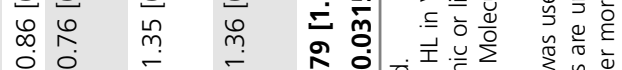

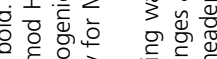

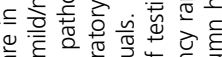

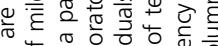

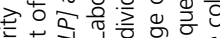

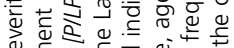

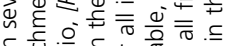

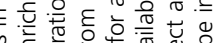

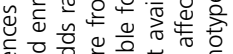
证

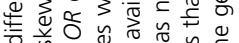

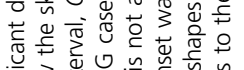

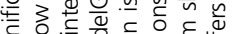
흔 는

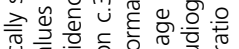

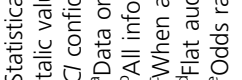


suggesting pathogenicity. Based on professional judgment, the ClinGen HL-EP ruled that PS4 overrides BA1.

When applying PS4, we need to choose appropriate cases and controls. Because mild hearing loss may be underdiagnosed without a formal audiology evaluation, we opted to compare cases versus population controls. Should results be significant in cases over population controls, they would be even more so in cases over unaffected controls.

We chose to use laboratory-contributed data for cases instead of relying on published reports to avoid publication biases. Negative results are difficult to get published. Some patients may be included in multiple studies. Large cohort studies do not generally provide detailed case-level data, and cases studies do not specify the total number of cases tested for the variant of interest.

In this study, we performed different types of case-control analyses. Although alleles were significantly enriched in cases with ORs of 1.3 (95\% CI 1.2-1.5, $Z=4.2, p<0.0001$ ) for $\mathrm{p}$. Met34Thr in Europeans (Table 2) and of 1.7 (95\% CI 1.5-1.9, $Z=8.1, p<0.0001$ ) for p.Val37Ile in Asians (Table 3), the ORs did not exceed 5 to satisfy PS4 according to ACMG/AMP guidelines, ${ }^{2}$ consistent with a previous meta-analysis. ${ }^{31}$ However, ORs of homozygote frequencies met PS4. Hence, comparing individual frequencies is more appropriate than comparing allele frequencies in case-control analyses to interpret variants associated with an autosomal recessive condition, because unaffected heterozygous carriers contributed the majority of alleles.

We observed a larger effect when including compound heterozygotes, which implied a higher penetrance of other pathogenic variants in GJB2 than p.Met34Thr and p.Val37Ile. Our data indicate that it is preferable to include compound heterozygotes in analyses. Because individual data are unavailable from population databases such as gnomAD, population carrier screening data are invaluable.

Ethnicity information is crucial in case-control studies, because different ethnicity compositions could confound results. In this study, we found significances were inflated when ethnicity information was disregarded. Proportions of p.Met34Thr and p.Val37Ile homozygotes varied significantly among laboratories due to different population compositions tested. Combining data from different laboratories while ignoring ethnicity information would bias toward the ethnic composition of the laboratory that contributed the most data. Stratifying patients by ethnicity significantly reduces the bias but decreases the sample size. Combining data from laboratories around the world allowed us to perform unbiased analysis with sufficient statistical power. Unfortunately, ethnicity information is not always submitted to testing laboratories, and some laboratories do not collect such information. Furthermore, ethnicity in gnomAD was inferred based on principal component analysis, which may differ from self-reported ethnicity; gnomAD tends to overestimate major populations as people with a self-reported mixed ethnic background would likely be counted toward one of the major populations. Therefore, we recommend laboratories collect ethnic information on the test requisition form and request patients and physicians to provide such information when ordering genetic tests.

\section{Genotype-phenotype correlation}

Variable expressivity of p.Met34Thr and p.Val37Ile has been reported. $^{16,32}$ The hearing loss may be unilateral or bilateral, from mild to profound, affecting different frequency ranges, even in individuals with the same genotype. P.Val37Ile has also been associated with pathopoieia ${ }^{33}$ and sudden hearing loss. $^{34}$ There have been over 100 publications reporting individuals with these variants. However, case-level genotype and phenotype data were limited. This multicenter study found over $96 \%$ of biallelic p.Met34Thr or p.Val37Ile cases with bilateral hearing loss including a small percentage with asymmetric presentations, $84 \%$ with mild to moderate hearing loss, and a majority with high-frequency loss, consistent with previous reports. ${ }^{35-38}$ Individuals with profound hearing loss may have an alternate etiology such as infection or old age. Unilateral or asymmetrical hearing loss may progress to bilateral uniform.

The majority of diagnosed cases in our multicenter cohort had a pediatric onset of hearing loss. The findings could be due to ascertainment bias, because most individuals undergoing genetic diagnosis are younger than 18 years old. Adults with mild hearing loss may not seek audiology evaluation or genetic testing. Progression of hearing loss was only reported in a small percentage of cases, because the progression was slow $^{15}$ and long-term follow-up information was unavailable.

We confirmed incomplete penetrance of these two variants. Three Asian p.Val37Ile homozygotes and two Ashkenazi Jewish p.Met34Thr compound heterozygotes were confirmed unaffected by audiology evaluation with the oldest known age at testing of 30 years (Table 5). We also identified 411 individuals with biallelic p.Met34Thr (189) or p.Val37Ile (238) via carrier testing. However, the penetrance could not be quantified based on our data, because they might be truly unaffected or underdiagnosed. It has been estimated that the penetrance of $\mathrm{p}$.Val37Ile homozygotes was $17 \%$ in children in China, ${ }^{16}$ but the number could vary by age, ethnicity, and the allele in trans in compound heterozygotes. Our data suggest that the penetrance is higher in p.Met34Thr or p.Val37Ile compound heterozygotes than in corresponding homozygotes.

The mechanism for incomplete penetrance and variable expressivity remains elusive. Environmental and genetic modifiers may play a role. It is unclear how many different haplotypes were involved and whether the phenotype is haplotype-dependent. The haplotype with c.-684_-675del in 5'UTR of NM_004004.5 (rs139514105, referred to as c.493del10) in cis with p.Met34Thr was confirmed in an affected sibpair from the $\mathrm{UK}^{8}$ and seven affected German individuals, ${ }^{39}$ but its frequencies in all affected and unaffected biallelic p.Met34Thr populations are unknown. This haplotype did not abolish GJB2 expression in cultured keratinocytes, which argues against a regulatory role of the noncoding 
Table 5 Unaffected p.Met34Thr or p.Val37lle homozygotes or compound heterozygotes confirmed by audiology evaluation

\begin{tabular}{lllllllll} 
Site & ID & Var 1 DNA & Var 1 AA & Var 2 DNA & Var 2 AA & Age tested & Family history & Ethnicity \\
\hline LMM & $56 \mathrm{~s}$ & c.109G $>$ A & p.Val37lle & c.109G $>A$ & p.Val37lle & 7 years old & Sibling & Asian \\
NTMC & 101 & c.109G $>$ A & p.Val37lle & c.109G $>A$ & p.Val37lle & 28 years old & No & Asian \\
NTMC & 102 & c.109G $>$ A & p.Val37lle & c.109G $>A$ & p.Val37lle & 30 years old & No & Asian \\
TAU & 13 & c.101T>C & p.Met34Thr & c.269T>C & p.Leu90Pro & Adult & Unknown & Ashkenazi//raqi Jewish \\
TAU & 14 & c.101T>C & p.Met34Thr & c.167delT & p.Leu56Argfs & Unknown & Unknown & Ashkenazi Jewish \\
\hline
\end{tabular}

Individuals without phenotypic information or audiology evaluation are not listed.

cis variant. ${ }^{8}$ Identification of the modifiers will not only help interpret the genetic findings but also point to effective therapeutic strategies for GJB2-related hearing loss, the most common form of hereditary hearing loss. Given the high allele frequency of these two variants in the population, further studies to identify modifiers through population genetic screening and phenotypic evaluation of biallelic individuals are warranted and feasible.

\section{Barriers to accurate variant interpretation}

Our experience in analyzing p.Met34Thr and p.Val37Ile in GJB2 revealed barriers to accurate variant interpretation. First, no phenotypic information was available in large population studies or population screening. Although we were able to achieve statistical significance with a large sample size, should hearing status in the general population be known, a much smaller sample size would be sufficient to provide the same level of statistical power. Second, incomplete ethnicity, family history, and clinical information from laboratories and publications diminished the usefulness of many cases. We would urge ordering physicians and testing laboratories to collect and share such information and editors and peer reviewers to encourage publication of detailed case-level information. Third, case information was biased toward published case reports usually by clinicians and genetic service centers. Although they provided detailed clinical information, matched control information was mostly unavailable. A systematic patient registry to document harmonized clinical and genetic information will overcome this. Finally, given the slow progressiveness and variable expressivity, we still could not accurately determine the penetrance of these variants in manifesting hearing loss and pinpoint the factors that influence the penetrance. Although age seems to be a factor, it could not explain everything. Long-term follow-up of individuals with these variants in prospective studies will illuminate in this area.

\section{Conclusion}

We conclude that the ACMG/AMP variant interpretation framework can be applied to variants with incomplete penetrance. Large and diverse sample sizes are required to overcome limitations and draw valid conclusions. The ClinGen HL-EP established a collaborative model of operation to collect case-level data, which allowed unbiased analysis of p.Met34Thr and p.Val37Ile, two controversial variants in
GJB2. Based on compelling statistical and supporting functional evidence, we conclude that the two GJB2 variants meet criteria to be classified as pathogenic for autosomal recessive sensorineural hearing loss, typically bilateral mild to moderate and slowly progressive over time. ${ }^{15}$ When these variants are identified in homozygosity or compound heterozygosity in GJB2 in cases with profound hearing loss, an alternate etiology should be investigated.

\section{SUPPLEMENTARY INFORMATION}

The online version of this article (https://doi.org/10.1038/s41436019-0535-9) contains supplementary material, which is available to authorized users.

\section{ACKNOWLEDGEMENTS}

We thank members of the ClinGen Hearing Loss Working Group who participated in the discussion on applying ACMG/AMP and $\mathrm{HL}$-specific rules to determine the classification of the p.Met34Thr and p.Val37lle variants. We thank Donglin Bai of the Schulich School of Medicine \& Dentistry, Western University for critical and constructive comments on the manuscript. This work was supported by National Institutes of Health/National Institute on Deafness and Other Communication Disorders (NIH/NIDCD) grants R03DC013866 and R01DC015052 (to J.S.); R01DC011835 (to K.B.A.); NIH/NINDS R01AR059049, NIH/ National Human Genome Research Institute (NHGRI) U01HG008666. and three other intramural grants (to K.Z.); a Grant-in-Aid for Clinical Research from the National Hospital Organization H27-NHOkankaku-02, Japan (to T.M.); Spanish Instituto de Salud Carlos III grants PI14/01162 (to I.d.C.) and PI14/0948 (to M.A.M.-P.); Regional Government of Madrid-Spain RAREGENOMICS-CAM grant B2017/BMD3721 (to M.A.M.-P.); and Plan Estatal de I+D+I 2013-2016 with cofunding from the European Regional Development Fund (to I.d.C. and M.A.M.-P.).

\section{CLINGEN HEARING LOSS WORKING GROUP}

Hela Azaiez ${ }^{32}$, Kevin T. Booth ${ }^{32}$, Richard J. Smith ${ }^{32}$, Anne B. Giersch ${ }^{33}$, Cynthia C. Morton ${ }^{33}$, Xue Z. Liu ${ }^{34}$, Mustafa Tekin ${ }^{34}$, Yu Lu $^{35}$, Huijun Yuan ${ }^{35}$, Hideki Mutai ${ }^{36}$, Lisa Schimmenti ${ }^{37}$

${ }^{32}$ University of lowa School of Medicine, Dongling Bai of University of Western Ontario in Canada, Alexander Chapin and Rong Mao of ARUP Laboratories, lowa City, IA, USA; ${ }^{33}$ Brigham and Women's Hospital, Hannie Kremer of Radboud University Medical Center in the Netherlands, Boston, MA, USA; ${ }^{34}$ University 
of Miami Miller School of Medicine, Miami, FL, USA; ${ }^{35}$ Southwest Hospital in China, Chongqing Shi, China; ${ }^{36}$ National Hospital Organization Tokyo Medical Center, Tokyo, Japan; ${ }^{37}$ Mayo Clinic, and Wenying Zhang of Cincinnati Children's Hospital Medical Center, Cincinnati, OH, USA; ${ }^{38}$ Present address: New York Genome Center, New York, NY 10013, USA;

${ }^{39}$ Present address: ConsulGene, LLC, Jacksonville, FL, USA; ${ }^{40}$ Present address: Department of Ophthalmology, University of Alabama at Birmingham School of Medicine, Birmingham, AL, USA

\section{DISCLOSURE}

J.S., A.M.O., H.D., J.J.A., M.L., K.Z., S.S.A., H.L.R., and A.N.A.T. worked for pay for service diagnostic laboratories providing genetic testing. H.P.K., S.G., R.M.-H., K.M., N.N., and A.W. worked for commercial laboratories providing genetic testing. The other authors declare no conflicts of interest.

Publisher's note: Springer Nature remains neutral with regard to jurisdictional claims in published maps and institutional affiliations.

\section{REFERENCES}

1. Wang NK, Chiang JPW. Increasing evidence of combinatory variant effects calls for revised classification of low-penetrance alleles. Genet Med. 2018 Nov 2; https://doi.org/10.1038/s41436-018-0347-3 [Epub ahead of print].

2. Richards S, Aziz N, Bale $S$, et al. Standards and guidelines for the interpretation of sequence variants: a joint consensus recommendation of the American College of Medical Genetics and Genomics and the Association for Molecular Pathology. Genet Med. 2015;17:405-424.

3. Oza AM, DiStefano MT, Hemphill SE, et al. Expert specification of the ACMG/AMP variant interpretation guidelines for genetic hearing loss. Hum Mutat. 2018;39:1593-1613.

4. Kelsell DP, Dunlop J, Stevens HP, et al. Connexin 26 mutations in hereditary non-syndromic sensorineural deafness. Nature. 1997:387:80-83.

5. Scott DA, Kraft ML, Carmi R, et al. Identification of mutations in the connexin 26 gene that cause autosomal recessive nonsyndromic hearing loss. Hum Mutat. 1998;11:387-394.

6. Scott DA, Kraft ML, Stone EM, et al. Connexin mutations and hearing loss. Nature. 1998;391:32.

7. Wilcox SA, Saunders $\mathrm{K}$, Osborn AH, et al. High frequency hearing loss correlated with mutations in the GJB2 gene. Hum Genet. 2000;106:399-405.

8. Houseman MJ, Ellis LA, Pagnamenta A, et al. Genetic analysis of the connexin-26 M34T variant: identification of genotype M34T/M34T segregating with mild-moderate non-syndromic sensorineural hearing loss. J Med Genet. 2001;38:20-25.

9. Cucci RA, Prasad S, Kelley PM, et al. The M34T allele variant of connexin 26. Genet Test. 2000;4:335-344.

10. Kelley PM, Harris DJ, Comer BC, et al. Novel mutations in the connexin 26 gene (GJB2) that cause autosomal recessive (DFNB1) hearing loss. Am J Hum Genet. 1998;62:792-799.

11. Abe S, Usami S, Shinkawa H, et al. Prevalent connexin 26 gene (GJB2) mutations in Japanese. J Med Genet. 2000;37:41-43.

12. Griffith AJ, Chowdhry AA, Kurima $K$, et al. Autosomal recessive nonsyndromic neurosensory deafness at DFNB1 not associated with the compound-heterozygous GJB2 (connexin 26) genotype M34T/167delT. Am J Hum Genet. 2000;67:745-749.

13. Feldmann $D$, Denoyelle $F$, Loundon $N$, et al. Clinical evidence of the nonpathogenic nature of the M34T variant in the connexin 26 gene. Eur J Hum Genet. 2004;12:279-284.

14. Pollak A, Skorka A, Mueller-Malesinska M, et al. M34T and V37I mutations in GJB2 associated hearing impairment: evidence for pathogenicity and reduced penetrance. Am J Med Genet A. 2007;143A:2534-2543.
15. Wu CC, Tsai $\mathrm{CH}$, Hung CC, et al. Newborn genetic screening for hearing impairment: a population-based longitudinal study. Genet Med. 2017;19:6-12.

16. Chai $Y$, Chen $D$, Sun $L$, et al. The homozygous p.V37I variant of GJB2 is associated with diverse hearing phenotypes. Clin Genet. 2015;87:350-355.

17. White TW, Deans MR, Kelsell DP, Paul DL. Connexin mutations in deafness. Nature. 1998:394:630-631.

18. Skerrett IM, Di WL, Kasperek EM, et al. Aberrant gating, but a normal expression pattern, underlies the recessive phenotype of the deafness mutant Connexin26M34T. FASEB J. 2004;18:860-862.

19. Palmada M, Schmalisch K, Bohmer C, et al. Loss of function mutations of the GJB2 gene detected in patients with DFNB1-associated hearing impairment. Neurobiol Dis. 2006;22:112-118.

20. Martin PE, Coleman SL, Casalotti SO, et al. Properties of connexin26 gap junctional proteins derived from mutations associated with non-syndromal heriditary deafness. Hum Mol Genet. 1999;8: 2369-2376.

21. Thonnissen $\mathrm{E}$, Rabionet R, Arbones ML, et al. Human connexin26 (GJB2) deafness mutations affect the function of gap junction channels at different levels of protein expression. Hum Genet. 2002;111: 190-197.

22. Bicego $M$, Beltramello $M$, Melchionda $S$, et al. Pathogenetic role of the deafness-related M34T mutation of Cx26. Hum Mol Genet. 2006:15:2569-2587.

23. Zonta F, Buratto D, Cassini $C$, et al. Molecular dynamics simulations highlight structural and functional alterations in deafness-related M34T mutation of connexin 26. Front Physiol. 2014;5:85.

24. D'Andrea P, Veronesi $V$, Bicego $M$, et al. Hearing loss: frequency and functional studies of the most common connexin26 alleles. Biochem Biophys Res Commun. 2002;296:685-691.

25. de Wolf E, van de Wiel J, Cook J, Dale N Altered CO2 sensitivity of connexin26 mutant hemichannels in vitro. Physiol Rep. 2016;4: e13038;1-12.

26. Maeda S, Nakagawa S, Suga M, et al. Structure of the connexin 26 gap junction channel at 3.5 A resolution. Nature. 2009:458:597-602.

27. Yilmaz A. Bioinformatic analysis of GJB2 gene missense mutations. Cell Biochem Biophys. 2015;71:1623-1642.

28. Bruzzone R, Veronesi V, Gomes D, et al. Loss-of-function and residual channel activity of connexin26 mutations associated with non-syndromic deafness. FEBS Lett. 2003;533:79-88.

29. Kim J, Jung J, Lee MG, et al. Non-syndromic hearing loss caused by the dominant cis mutation R75Q with the recessive mutation V37I of the GJB2 (Connexin 26) gene. Exp Mol Med. 2015;47:e169.

30. Chen $Y$, Hu L, Wang $X$, et al. Characterization of a knock-in mouse model of the homozygous p.V37I variant in Gjb2. Sci Rep. 2016;6:33279.

31. Chan DK, Chang KW. GJB2-associated hearing loss: systematic review of worldwide prevalence, genotype, and auditory phenotype. Laryngoscope. 2014;124:E34-53.

32. Lameiras AR, Goncalves $A C$, Santos $R$, et al. The controversial $p$. Met34Thr variant in GJB2 gene: two siblings, one genotype, two phenotypes. Int J Pediatr Otorhinolaryngol. 2015;79:1316-1319.

33. Dai ZY, Sun BC, Huang SS, et al. Correlation analysis of phenotype and genotype of GJB2 in patients with non-syndromic hearing loss in China. Gene. 2015;570:272-276.

34. Chen $K$, Sun L, Zong L, et al. GJB2 and mitochondrial $12 S$ rRNA susceptibility mutations in sudden deafness. Eur Arch Otorhinolaryngol. 2016;273:1393-1398.

35. Lim LH, Bradshaw JK, Guo Y, et al. Genotypic and phenotypic correlations of DFNB1-related hearing impairment in the Midwestern United States. Arch Otolaryngol Head Neck Surg. 2003;129:836-840.

36. Lee KH, Larson DA, Shott G, et al. Audiologic and temporal bone imaging findings in patients with sensorineural hearing loss and GJB2 mutations. Laryngoscope. 2009;119:554-558.

37. Kenna MA, Feldman HA, Neault MW, et al. Audiologic phenotype and progression in GJB2 (Connexin 26) hearing loss. Arch Otolaryngol Head Neck Surg. 2010;136:81-87.

38. Jiang $Y$, Huang $S$, Deng $T$, et al. Mutation spectrum of common deafnesscausing genes in patients with non-syndromic deafness in the Xiamen Area, China. PLoS ONE. 2015:10:e0135088.

39. Zoll $B$, Petersen L, Lange $K$, et al. Evaluation of Cx26/GJB2 in German hearing impaired persons: mutation spectrum and detection of disequilibrium between M34T (c.101T>C) and -493del10. Hum Mutat. 2003:21:98 
${ }^{1}$ Department of Pathology, Brigham and Women's Hospital, Harvard Medical School, Boston, MA, USA. ${ }^{2}$ Harvard Medical School Center for Hereditary Deafness, Boston, MA, USA. ${ }^{3}$ Laboratory for Molecular Medicine, Partners HealthCare Personalized Medicine, Cambridge, MA, USA. 'Department of Otolaryngology and Communication Enhancement, Boston Children's Hospital, Harvard Medical School, Boston, MA, USA. ${ }^{5}$ Servicio de Genetica, Hospital Universitario Ramon y Cajal, IRYCIS, Madrid, Spain. ${ }^{6}$ Centro de Investigacion Biomedica en Red de Enfermedades Raras (CIBERER), Madrid, Spain. ${ }^{7}$ Cincinnati Children's Hospital Medical Center, Cincinnati, OH, USA. ${ }^{8}$ Division of Hearing and Balance Research, National Institute of Sensory Organs, National Hospital Organization Tokyo Medical Center, Tokyo, Japan. ${ }^{9}$ University of North Carolina, Chapel Hill, NC, USA. ${ }^{10}$ Counsyl, South San Francisco, CA, USA. ${ }^{11}$ EGL Genetics/Department of Human Genetics, Emory University School of Medicine, Atlanta, GA, USA. ${ }^{12}$ Certer for Medical Genetics, Guangdong Women and Children Hospital, Guangzhou, Guangdong, China. ${ }^{13}$ Dor Yeshorim, Committee for Prevention of Jewish Genetic Diseases, Brooklyn, NY, USA. ${ }^{14}$ The Children's Hospital of Philadelphia, Philadelphia, PA, USA. ${ }^{15}$ The University of Pennsylvania Perelman School of Medicine, Philadelphia, PA, USA. ${ }^{16}$ Department of Obstetrics and Gynecology, The Chinese University of Hong Kong, Hong Kong, China. ${ }^{17}$ Department of Medical Research, Taipei Veterans General Hospital, Taipei, Taiwan. ${ }^{18}$ Department of Otolaryngology-Head and Neck Surgery, Taipei Veterinary Hospital, Taipei, Taiwan.

${ }^{19}$ School of Medicine, National Yang-Ming University, Taipei, Taiwan. ${ }^{20}$ Raphael Recanati Genetic Institute, Rabin Medical CenterBeilinson Hospital, Petach Tikva, Israel. ${ }^{21}$ Pediatric Genetics Clinic, Schneider Children's Medical Center of Israel, Petach Tikva, Israel. ${ }^{22}$ Sackler Faculty of Medicine, Tel Aviv University, Tel Aviv, Israel. ${ }^{23}$ Felsenstein Medical Research Center, Petach Tikva, Israel.

${ }^{24}$ Department of Biostatistics, Fairbanks School of Public Health and School of Medicine, Indiana University, Indianapolis, IN, USA. ${ }^{25}$ Danek Gartner Institute of Human Genetics, Sheba Medical Center, Tel Hashomer, Israel. ${ }^{26}$ Dor Yeshorim, Committee for Prevention of Jewish Genetic Diseases, Jerusalem, Israel. ${ }^{27}$ Integrated Genetics, Laboratory Corporation of America ${ }^{\circledR}$ Holdings, Westborough, MA, USA. ${ }^{28}$ Integrated Genetics, Laboratory Corporation of America ${ }^{\circledR}$ Holdings, Research Triangle Park, NC, USA. ${ }^{29}$ The Broad Institute of MIT and Harvard, Cambridge, MA, USA. ${ }^{30}$ Center for Genomic Medicine, Massachusetts General Hospital, Boston, MA, USA. ${ }^{31} \mathrm{Al}$ Jalila Children's Specialty Hospital, Dubai, UAE 